\title{
Robust Tracking of Piecewise Linear Trajectories with Binary Sensor Networks
}

\author{
Er-wei Bai ${ }^{\text {a,b }}$, Henry Ernest Baidoo-Williams ${ }^{\text {a }}$, Raghuraman Mudumbai ${ }^{\text {a }}$, \\ Soura Dasgupta ${ }^{a}$ \\ ${ }^{a}$ Department of Electrimathcal and Computer Engineering, University of Iowa, Iowa City, IA 52242. Phone 319-335-5949 \\ b School of Electronics, Electrimathcal Engineering and Computer Science, Queen's University, Belfast, UK
}

\begin{abstract}
We present a novel approach to the problem of tracking objects moving along smooth trajectories using a network of simple inexpensive binary sensors. Specifimathcally, we consider object trajectories that can be well-approximated by piecewise linear curves and sensors that can only detect whether an object is in their sensing range. We start by considering objects moving along straight-line trajectories with an unknown speed and show that such objects can be tracked using the measurements of just three generically placed binary sensors whose sensing ranges intersect the trajectory. Next, we present an asymptotic analysis that shows that a trajectory consisting of a finite number of straight line segments can be recovered with high probability using an arbitrarily low spatial density of sensors in the limit when the area to be covered gets larger and larger. We also present efficient algorithms that effectively recover piecewise linear trajectories. Finally we present analysis and simulations to demonstrate the high tracking accuracy of our approach and its robustness to sensing errors.
\end{abstract}

Key words: Tracking, localization, binary sensor network.

\section{Introduction}

Localization and tracking of the path of an object are basic problems in many applications, e.g., surveillance, border monitoring, rescue, security, agriculture and many others. Fundamentally, these problems involve estimation of the position and trajectory of objects of interest using the observations of a network of sensors, [21], [20].

The reliability of a sensor's observations is a function of the strength of the sensor's interaction with the object they track [1], which in turn generally depends on how close the sensors are to their target. This proximity requirement is especially stringent for sensing applications where signal absorption by the medium is large. For example, the strength of gamma rays emanated by a radioactive source, in the presence of structures that act as shielding material [13], exponentially declines with distance. This suggests that in

\footnotetext{
^ Corresponding author Er-Wei Bai

Email addresses: erwei@engineering. uiowa.edu (Er-wei Bai), henry-baidoo-williams@uiowa.edu ( Henry Ernest Baidoo-Williams),

rmudumbai@engineering . uiowa.edu (Raghuraman Mudumbai), dasgupta@engineering. uiowa.edu (Soura Dasgupta).
}

order to continuously track a moving object, it is necessary to deploy a large number of sensors throughout an area of interest in order to avoid "coverage gaps"; high tracking accuracy under this setting would require dense deployment of sensors with correspondingly high cost and complexity.

This paper considers the possibility of significantly reducing the cost and complexity of the tracking problem by exploiting the continuity and smoothness of the trajectories of real physical objects. Specifically, we consider tracking using a network of simple binary sensors. The density of sensor deployment, defined as the total number of sensors per unit area, only needs to be large enough to ensure that the speed and bearing of the object remains roughly constant over multiple sensor observations. This is much easier to satisfy than requiring that the object remain within range of at least one sensor at all times.

We also allow individual sensors to be simple, noisy and vulnerable to drift and calibration errors, and rely on statistical robustness by combining the observations of many sensors. This is in keeping with the basic philosophy of large-scale sensor networks [3] that favors distributed networks of simple, inexpensive, disposable sensors to a small number of complex, expensive ones. With noisy sensors, it is also possible to radically quantize observations [2] with little sacri- 
fice in performance.

In fact, the sensors we consider are binary sensors, that can only detect if an object is within in their sensing range. Effectively, this is a case of one-bit signal quantization. The observations of binary sensors tracking a moving object continuously, can be efficiently summarized by recording the time-stamps of the instants at which a moving object enters and leaves the sensor's detection range. This in turn allows sensors to communicate their observations to a fusion center with very little overhead (just two time-stamps for each object passing through a sensor's range). Because the communication requirements are so minimal, we assume in this paper that all sensor observations are available at a centralized fusion center and therefore we do not consider the possibility of further reducing overheads using distributed algorithms.

Related Work. Binary sensors represent a convenient and powerful abstraction for a variety of physical sensors, and different types of binary sensors have been widely studied in the literature on localization and tracking [4-11, 14, 17-19]. One type of binary sensor network tries to use the geometry of the network [19] and considers the network as a connected graph to aid detection. The work in [10] considers heterogeneous binary sensors with different performance and cost. The purpose is to analyze the best proportion of sensors in each class to tradeoff performance with cost. Some assume probabilistic models for object movement and/or for the received signal strength $[7,9,11,14,17]$ and develop detection algorithms based on these models. Others additionally assume that each sensor [19] has the ability to sense if the object is approaching or moving away.

Many of the existing studies rely on the density of the sensors being large enough to essentially guarantee blanket coverage of the region of interest [5], [4]. The idea is that if the density is high enough, every movement of the object lies in the sensing range of several sensors and the object's position and trajectory can be pinned down with a higher degree of accuracy because the intersection of sensing ranges of multiple sensors is much smaller than the sensing area of any single sensor. Clearly, a higher density implies that the sensing ranges of more sensors intersect which results in a smaller intersecting area or a more accurate estimate. In fact, as shown in [5] the estimation accuracy is of the order $1 / \rho$, where $\rho$ is the sensor density assuming all sensors have the same sensing range.

Contributions. In this paper, we ask the following fundamental question: how many binary sensors are needed to track an object moving in an unknown piecewise linear trajectory with unknown speed? We are motivated by (a) the observation that inertial and other dynamical constraints restrict most trajectories to be smooth, and (b) smooth trajectories can be arbitrarily well approximated by piecewise linear trajectories. These assumptions are reasonable in some applications. Consider an example of monitoring radioactive material smuggling at a sea port of entry which consists of a number of piecewise linear roads from unloading cargoes to the exit of the port. Our main contributions are as follows.

- We show that three generically placed sensors can uniquely determine almost all linear trajectories that intersect with their sensing range. This naturally extends to piecewise linear trajectories that are trajectories containing a finite number of connected line segments.

- We also show that sensors deployed randomly with vanishingly small densities can uniquely determine a straight line trajectory with high probability. More generally, for smooth trajectories, the required sensor density for accurate tracking is determined only by the number of piecewise linear segments needed to sufficiently approximate the trajectory, and this density can be much smaller than what is required to achieve blanket coverage.

- The geometric problem of determining the points of intersection of a straight-line trajectory with the coverage area of a binary sensor involves solving a set of non-linear equations. However, we present a reformulation of the tracking problem that involves a constrained set of linear equations and allows a simple least-squares solution.

- Based on the above reformulation into linear equations, we present a practical, computationally efficient algorithm to solve the piecewise linear trajectory estimation problem.

- We conduct an extensive robustness analysis of the algorithm under departures from the idealizing assumptions. Specfically we show that the algorithm can tolerate uncertainty in the sensing ranges. Further we show that it can track objects that do not travel on a precise piecewise linear trajectory.

- Finally, we present an extensive set of simulations to illustrate the performance of our proposed approach and its robustness to sensor errors and uncertainties.

Our presentation in this paper proceeds in a step-by-step fashion. First we consider the simple and idealized setting of tracking objects moving in straight-line trajectories with known speeds and homogenous noise-free sensors. While these assumptions are of course unrealistic, this initial analysis allows us to present key geometric intuitions concisely, and also to present a simple trajectory estimation algorithm that involves solving a set of linear equations with constraints. We then show that this algorithm can be used to estimate arbitrary trajectories that can be approximated with piecewise linear segments, and extended to remove restrictions of known target speed and ideal sensors. Practical sensors may deviate from the model of an ideal binary sensor in many different ways e.g. measurement noise, calibration error, non-circular sensing range and possibility of sensor failure i.e. misses or false positives; our analysis shows the robustness of our approach to calibration error, and we present simulations to demonstrate that we are able to tolerate other types of disturbances.

The organization of the paper is as follows. Section 2 describes the problem formulation. Section 3 considers a target moving with known speed, and describes a simple al- 
gorithm to estimate piecewise linear trajectories. Section 4 takes these basic results and extends them to the case where the target speed is unknown. Section 5 addresses robustness issues associated with uncertainties in the sensing range of the sensors. It also shows that the algorithm can track a source that does not travel on an exact piecewise linear trajectory. Section 6 quantifies the required density of sensors needed to track linear trajectories. Section 7 presents simulation results. Section 8 concludes.

\section{Problem setup}

Consider a region of interest $\mathcal{D} \subset \mathbb{R}^{2}$ over which we would like to track a moving object, and a binary sensor network of $N$ sensors which are deployed over the region.

Let $\left(x_{i}, y_{i}\right)$ 's, $i \in\{1,2, \ldots, N\}$, denote the locations of the sensors. Each sensor continuously monitors the received signal strength of the object of interest and compares it with a prescribed threshold; if the signal at any instant is larger (smaller) than the threshold, the sensor determines that the object's position is inside (outside) its sensing range. As noted earlier, the entire measurement history of a binary sensor can be fully summarized as a set of time-stamps of the instants when the object enters or leaves the sensing region of the sensor.

In the derivation, We first assume here that all sensors are homogeneous and ideal, i.e. that all sensors have the same nominal sensing range $r>0$. Notice that $r$ is the supremum of the distances from the homogeneous sensor centers $\left(x_{i}, y_{i}\right)$ such that the received signal strength is strictly greater than the set threshold. This is of course an ideal case; in practice each sensor has a sensing range $r+\Delta_{i}$, $i \in\{1,2, \ldots, N\}$, that deviates from the nominal range because of calibration errors or model uncertainties. To deal with these uncertainties, more realistic assumptions will be clearly stated in sections 5 when imperfection is introduced.

\section{Known speed}

Consider an object that moves in a straight line $L$ in the Cartesian coordinate system with a known speed $s$. Suppose the object passes through the sensing ranges of three binary sensors with "on" and "off" times (i.e. the time-stamps of the entry and exit time instants) $\tau_{i 1}$ and $\tau_{i 2}, i \in\{1,2,3\}$ respectively. Since the time interval $\tau_{i 2}-\tau_{i 1}$ and the speed $s$ are available, the chord lengths produced by the line in the three small circles can be calculated as $2 d_{i}=s\left(\tau_{i 2}-\right.$ $\left.\tau_{i 1}\right), i \in\{1,2,3\}$. The following fact is self-evident.

Lemma 3.1 A line intersects a circle of radius $r$ with the chord lengths $2 d$, if and only if the line is a tangent to the concentric circle of radius $\sqrt{r^{2}-d^{2}}$.

Next we consider a geometric result that appeals to the notion of homothetic centers. Specifically, the homothetic

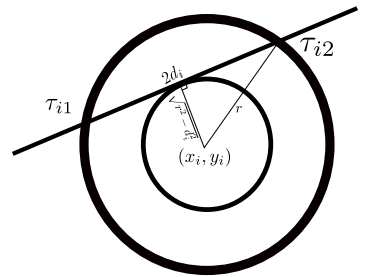

Fig. 1. Geometric illustration of lemma 3.1.

centers of two geometric similar figures are defined as points from which both the figures can be seen as a dilation/contraction of one another [15]. Thus, the internal and external homothetic centers for two circles are shown in Figure 2 (a). as the points of intersection of the internal and external tangents respectively.

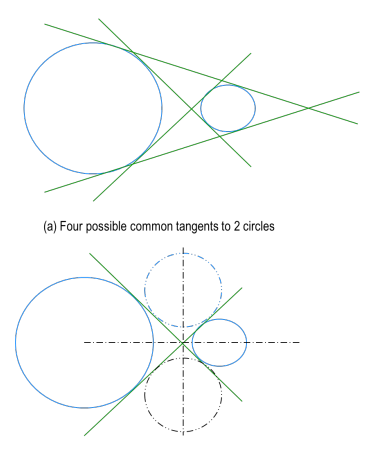

(c) 2 circles with two common intermal tangents

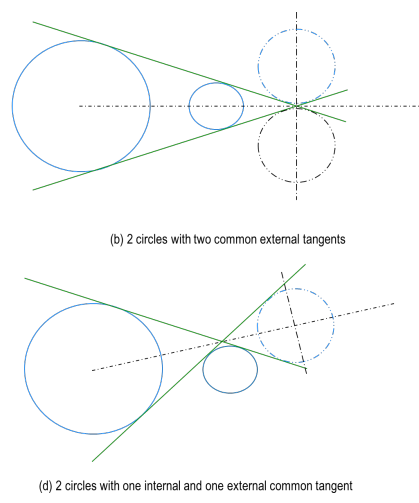

(d) 2 circles with one intermal and one extermal common tangent

Fig. 2. Illustration of homothetic center of two circles.

Lemma 3.2 Consider three non-overlapping circles with non-collinear centers. Suppose there are two distinct lines that are tangents to all three circles. Then the center of one of the circles must be on a line that is perpendicular to the line connecting the centers of the other two circles and passes through one of their homothetic centers.

Proof: For two non-overlapping circles, there are four possible shared tangent lines as shown in Figure 1(a). We have three possibilities where the center of the third circle is noncollinear with the first two centers and the third circle shares two tangent lines with both of the first two circles. The shared tangent lines could be one of the following:

(1) Two external tangents as in Figure 1 (b) if the first two circles have different radii.

(2) Two internal tangent lines as in Figure 1 (c)

(3) A pair of lines, one external and one internal tangent as in Figure 1 (d). Note, however, that this case is exactly the same as in Figure 1(c) by swapping the dashed circle with the solid one.

In view of the last point only the first two cases need to be considered. In both cases consider the circle depicted by dashed lines. As it is tangent to both external tangent lines, the center of this circle is on the line that is perpendicular to the line connecting the centers of the first two circles. Also 
the same line has to pass through the homothetic center [15]. This completes the proof.

Using this result, we now consider the question of whether the line representing the object trajectory is generically, uniquely determined by a set of three chord lengths, $2 d_{i}$, $i \in\{1,2,3\}$, from the sensing regions of three sensors.

Theorem 3.1 Consider a straight line $L$, and a set of three points $\left(x_{i}, y_{i}\right), i \in\{1,2,3\}$ that obey the following:

(i) The $\left(x_{i}, y_{i}\right), i \in\{1,2,3\}$ are randomly and uniformly placed in $\mathcal{D} \subset \mathbb{R}^{2}$.

(ii) The circles with radius $r$ centered on $\left(x_{i}, y_{i}\right)$ are nonoverlapping.

(iii) The straight line L intersects these three circles with chord lengths $2 d_{i}$.

Then the $\left(x_{i}, y_{i}\right), r$ and $d_{i}$ uniquely determine the line $L$, with probability 1 .

Proof: Because of Lemma 3.1, the chord lengths $2 d_{i}$ define three circles of radii $\sqrt{r^{2}-d_{i}^{2}}$, respectively centered at the points $\left(x_{i}, y_{i}\right)$, to which the line is simultaneously tangent. Conditioned on (i-iii), with probability 1 , these circles will have non-collinear centers and the center of no circle will be on the line that (a) is perpendicular to the line connecting the centers of other two circles and (b) passes through a homothetic center of the latter. Then from Lemma 3.2 this line is uniquely determined from $\left(x_{i}, y_{i}\right), r$ and $d_{i}$ with probability 1 .

If an object travels with known speed on a straight line that intersects the sensing ranges of three generically placed circles, then the chord lengths $2 d_{i}$ can be determined from $s\left(\tau_{i 2}-\tau_{i 1}\right)$. Thus, Theorem 3.1 proves that such a line can be uniquely determined with probability 1 .

\subsection{Efficient numerical solutions}

Theorem 3.1 shows that given the chord length measurements of three binary sensors, a straight line trajectory can be uniquely determined with probability 1 . We now consider the problem of designing an algorithm to estimate the trajectory from the measurements. One obvious possibility is to calculate the intersecting points of the line and circles by directly solving $a x+b y+c=0$ jointly with

$$
\left(x-x_{i}\right)^{2}+\left(y-y_{i}\right)^{2}=r^{2}, \quad \forall i \in\{1,2,3\} .
$$

Note that this is a nonlinear (quadratic) equation in the $a, b, c$ coefficients. More importantly, this direct procedure is not easily generalizable to the case of unknown speed $s$, or unknown sensing ranges. We present here an alternative method that is accurate yet practical and efficient, and which is also easily generalized to more realistic sensing models tackled in subsequent sections.

We start by noting that the trajectory estimation problem can be reduced to the problem of finding a shared tangent line to three circles of radius $\sqrt{r^{2}-d_{i}^{2}}$ centered at $\left(x_{i}, y_{i}\right)$, $i \in\{1,2,3\}$ respectively, where $2 d_{i}=\left(\tau_{i 2}-\tau_{i 1}\right) s$. First, we redefine the parameters of the line to $\alpha x+\beta y+\gamma=0$, where $\alpha=\frac{a}{\sqrt{a^{2}+b^{2}}}, \beta=\frac{b}{\sqrt{a^{2}+b^{2}}}$ and $\gamma=\frac{c}{\sqrt{a^{2}+b^{2}}}$. Observe that the distance of the line $\alpha x+\beta y+\gamma=0$ to the centers $\left(x_{i}, y_{i}\right)$ are $\left|\alpha x_{i}+\beta y_{i}+\gamma\right|=\sqrt{r^{2}-d_{i}^{2}}, i \in\{1,2,3\}$, or in matrix form, for particular sign choices:

$$
\underbrace{\left[\begin{array}{lll}
x_{1} & y_{1} & 1 \\
x_{2} & y_{2} & 1 \\
x_{3} & y_{3} & 1
\end{array}\right]}_{A_{3}}\left[\begin{array}{l}
\alpha \\
\beta \\
\gamma
\end{array}\right]=\underbrace{\left[\begin{array}{l} 
\pm \sqrt{r^{2}-d_{1}^{2}} \\
\pm \sqrt{r^{2}-d_{2}^{2}} \\
\pm \sqrt{r^{2}-d_{3}^{2}}
\end{array}\right]}_{e_{3}}
$$

subject to $\alpha^{2}+\beta^{2}=\frac{a^{2}}{a^{2}+b^{2}}+\frac{b^{2}}{a^{2}+b^{2}}=1$. The solution can be easily calculated by for instance the least squares solution

$$
\begin{gathered}
{[\alpha, \beta, \gamma]^{T}=\left(A_{3}^{T} A_{3}\right)^{-1} A_{3}^{T} e_{3}} \\
\text { subject to } \alpha^{2}+\beta^{2}=1 .
\end{gathered}
$$

Note that the expression in (3.2) is more complicated than it needs to be; for 3 sensors $A_{3}$ is a square matrix, hence its pseudo-inverse in (3.2) can be replaced with an inverse, i.e. (3.2) can be simplified to

$$
[\alpha, \beta, \gamma]^{T}=A_{3}^{-1} e_{3}
$$

We will, however, work with the expression in (3.2) which unlike (3.4) generalizes in a straight-forward way to arbitrary number of sensors.

There are four solutions (there are eight total possible combinations of the \pm signs in (3.1) from which we obtain four distinct sets of equations of the form (3.2)). Under the conditions of Theorem 3.1 we know that with probability 1 , only one solution exists, i.e., only one of the four solutions from the distinct combinations of the \pm signs satisfies $\alpha^{2}+\beta^{2}=1$. This suggests a simple algorithm for finding the trajectory that we outline next.

\subsection{Tracking algorithm for piecewise linear trajectories}

As noted earlier, we consider in this paper a class of trajectories that can be well approximated as piecewise linear with piecewise constant speeds i.e. the trajectory can be modeled as consisting of multiple straight line constant speed segments. This is a reasonable model for several practical tracking applications. Thus for instance, a container truck 
in a busy port, given the constraints of traffic laws and the inertia and turning radius limitations of large trucks, can be well- modeled as moving with roughly constant speeds over short time intervals with occasional turns possibly accompanied by speed changes.

We now show how to extend our previous analysis of straight line trajectories to this more general case. The basic idea is that there is a change of direction and speed whenever there does not exist a line consistent with the observed entry and exit time-stamps of the three most recently triggered sensors, i.e. whenever (3.2) and (3.3) do not have any solution.

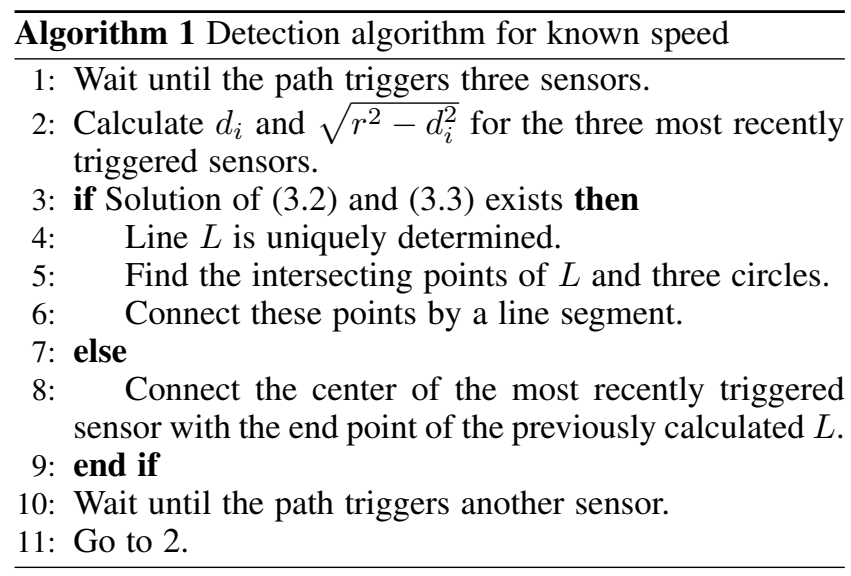

\section{Unknown speeds}

The preceding, Algorithm 1, assumes that the speed of the object is known at all times. This assumption permits us to determine chord lengths from time-stamps. In addition, it also assumes that the sensing ranges of different sensors are non-overlapping. We now show how our approach can be extended to relax these assumptions. The basic idea is to work with ratios of chord lengths. Over short distances where the constant (though unknown) speed approximation holds, the chord length ratios is equal to the corresponding ratios of time durations. The time durations can be calculated from the time-stamp measurements of the binary sensors.

We start by noting that from Theorem 3.1, even when the object speed is unknown, if we know that a line $L$ is tangent to three circles of known radii, then barring pathologies, this line will be unique. So in the following, we may assume that the line will produce at least one non-zero chord, say $\left(\tau_{12}-\tau_{11}\right) s \neq 0$.

At this point it is convenient to introduce some additional notation for the chord lengths. Specifically, let $l_{i}$ denote the "transition lengths" between the points where the trajectory enters or exits the sensing region of some sensor. This notation is illustrated in Figure 3. Note that the "chord lengths" $d_{i}$ in Figure 1 are related to $l_{i}$ as $2 d_{i}=l_{2 i-1}, i \in\{1,2,3\}$. now, even without knowing the speed, the time intervals $\tau_{i 2}-\tau_{i 1}$ and the chord length ratios can be calculated from the measured time stamps as:

$$
\frac{l_{i}}{l_{1}}=\left\{\begin{array}{l}
\frac{\left(\tau_{21}-\tau_{12}\right) s}{\left(\tau_{12}-\tau_{11}\right) s}=\frac{\tau_{21}-\tau_{12}}{\tau_{12}-\tau_{11}}, i=2 \\
\frac{\left(\tau_{22}-\tau_{21}\right) s}{\left(\tau_{12}-\tau_{11}\right) s}=\frac{\tau_{22}-\tau_{21}}{\tau_{12}-\tau_{11}}, i=3 \\
\frac{\left(\tau_{31}-\tau_{22}\right) s}{\left(\tau_{12}-\tau_{11}\right) s}=\frac{\tau_{31}-\tau_{22}}{\tau_{12}-\tau_{11}}, i=4 \\
\frac{\left(\tau_{32}-\tau_{31}\right) s}{\left(\tau_{12}-\tau_{11}\right) s}=\frac{\tau_{32}-\tau_{31}}{\tau_{12}-\tau_{11}}, i=5
\end{array}\right.
$$

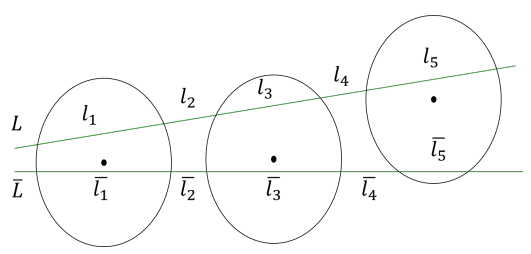

Fig. 3. Illustration of transition lengths $l_{i}$.

Theorem 4.1 Consider a straight line L, and a set of three points $\left(x_{i}, y_{i}\right), i \in\{1,2,3\}$ that obey the following:

(i) The $\left(x_{i}, y_{i}\right), i \in\{1,2,3\}$ are placed randomly and uniformly in $\mathcal{D} \subset \mathbb{R}^{2}$.

(ii) They are distinct.

(iii) The straight line $L$ intersects the three circles, each of radius $r$, centered on $\left(x_{i}, y_{i}\right)$, with $l_{i} \neq 0, i \in\{1, \cdots, 5\}$, depicted in Figure 3.

Then the $\left(x_{i}, y_{i}\right), r$ and the ratios $\frac{l_{i}}{l_{1}}, i \in\{2,3,4,5\}$ uniquely determine the line $L$, with probability 1 .

Before presenting the proof, we first provide 2 lemmas. Notice that through a rotation and translation, if need be, it is always possible to assume without loss of generality that the (unknown) line coincides with the horizontal axis and the first circle intersects the line at $\left(l_{1}, 0\right)$ as shown in Figure 3(A). This is possible because rotation and translation of the entire space $\mathcal{D} \in \mathbb{R}^{2}$ preserves distances from the sensors to $L$. We can therefore rotate $\mathcal{D} \in \mathbb{R}^{2}$ such that $L$ coincides with the horizontal axis and translate for the first sensors intersection with $L$ to be at any arbitrary point $\left(x_{a}, 0\right)$ of our choosing. We however maintain the notation for the sensor centers as $\left(x_{i}, y_{i}\right)$ in the transformed space to preserve clarity.

Lemma 4.1 Given an (unknown) line $y=k x+b,\{k=$ $\left.-\frac{\alpha}{\beta}, b=-\frac{\gamma}{\beta}\right\}$, which intersects 3 circles of radius $r$ as described before, suppose the sensors are placed randomly and uniformly in $\mathcal{D} \subset \mathbb{R}^{2}$. Then, with probability one, the lengths

$$
l_{i} \neq l_{j}, \text { for } i \neq j,\{i, j\} \in\{1, \cdots, 5\}
$$

Proof: We only show that $l_{i} \neq l_{j},\{i, j\} \in\{1,2,3\}$. The proof of the other cases are identical. Suppose $l_{2} \doteq l_{1}$, the center of the second circle has to be on the dashed red circle 
with radius $r$ centered at $\left(2 l_{1}, 0\right)$ as shown in Figure 4(A), i.e, on a one dimensional manifold in a 2-dimensional space. Suppose $l_{3}=l_{1}$, the center of the second circle has to be on the lines of $y= \pm \sqrt{r^{2}-\left(\frac{l_{1}}{2}\right)^{2}}$ which are again two 1dimensional manifolds in a 2-dimensional space as shown in Figure 4(B). For $l_{3}=l_{2}$, the center of the second circle has to satisfy

$x=l_{1}+l_{2}+\frac{l_{2}}{2}=f_{x}\left(l_{2}\right)$

$y= \pm \sqrt{r^{2}-\left(\frac{l_{2}}{2}\right)^{2}}= \pm f_{y}\left(l_{2}\right)$

which are two line segments, $0 \leq l_{2} \leq 2 r$, in a 2dimensional space as shown in Figure 4(C). Each of these 1-dimensional manifolds are sets of measure zero in 2dimensional space. Since the sensors are randomly and uniformly placed, the probability that the second center lies on any of the above manifolds is zero. This completes the proof.

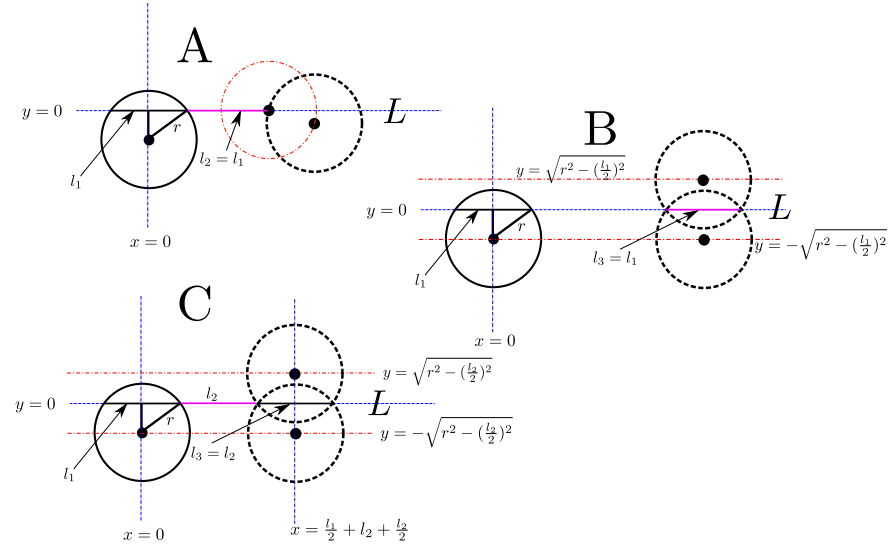

Fig. 4. (A) Depicts the case of $l_{1}=l_{2}$. (B) Depicts the case of $l_{1}=l_{3}$. (C) Depicts the case of $l_{2}=l_{3}$.

We now provide the second lemma. For this lemma, again without loss of generality, we may assume that the centers of the first two circles are at the origin and $\left(x_{2}, 0\right)$ for some $x_{2}>0$ respectively.

Lemma 4.2 Consider the setting depicted in Figure 3. Suppose all circles have radius $r$, the first two are centered at $(0,0)$ and $\left(x_{2}, 0\right)$ respectively, and the line in the figure is labeled as $L: y=k x+b,\left\{k=-\frac{\alpha}{\beta}, b=-\frac{\gamma}{\beta}\right\}$. Then, there exist only a finite number of lines that could intersect the first two circles with the same length ratios $\frac{l_{i}}{l_{1}}, i \in\{2,3\}$.

Proof: Consider a line $\bar{L}$ that intersects the first two circles. For the moment, assume that the line has the form $y=k x+b$ for some $k$ and $b$. We will find necessary conditions for $k$ and $b$ so that the line could intersect these two circles with the given length ratios. Suppose the points of intersection of the line $\bar{L}$ and the circles, moving from left to right in ascending order of $i$ are $\left(\bar{v}_{i}, \bar{u}_{i}\right)$ as shown in Figure 5 .

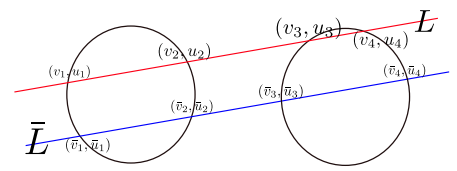

Fig. 5. Depiction of intersection points of $\bar{L}$ and $L$ with sensors

For $i \in\{1,2,3,4\}$ these obey:

$$
\bar{u}_{i}^{2}+\left(k \bar{u}_{i}+b\right)^{2}=r^{2},\left(\bar{u}_{i}-x_{2}\right)^{2}+\left(k \bar{u}_{i}+b\right)^{2}=r^{2} .
$$

This implies that with $\delta=\left(1+k^{2}\right)\left(b^{2}-r^{2}\right)$,

$$
\begin{aligned}
& \bar{v}_{1}=\frac{-k b-\sqrt{b^{2} k^{2}-\delta}}{1+k^{2}}, \\
& \bar{u}_{1}=k \bar{v}_{1}+b
\end{aligned}
$$

$$
\begin{aligned}
& \bar{v}_{2}=\frac{-k b+\sqrt{b^{2} k^{2}-\delta}}{1+k^{2}}, \\
& \bar{u}_{2}=k \bar{v}_{2}+b
\end{aligned}
$$

and

$$
\begin{aligned}
& \bar{v}_{3}=\frac{-\left(k b-x_{2}\right)-\sqrt{\left(b k-x_{2}\right)^{2}-\delta}}{1+k^{2}}, \\
& \bar{u}_{3}=k \bar{v}_{3}+b, \\
& \bar{v}_{4}=\frac{-\left(k b-x_{2}\right)+\sqrt{\left(b k-x_{2}\right)^{2}-\delta}}{1+k^{2}}, \\
& \bar{u}_{4}=k \bar{v}_{4}+b
\end{aligned}
$$

Define, as also depicted in Figure 3 and Figure 5,

$$
\bar{l}_{i}=\left\|\left(\begin{array}{l}
\bar{u}_{i+1} \\
\bar{v}_{i+1}
\end{array}\right)-\left(\begin{array}{c}
\bar{u}_{i} \\
\bar{v}_{i}
\end{array}\right)\right\|, i \in\{1,2,3\} .
$$

If $\bar{L}$ intersects the first two circles with the same length ratios as $L$, it follows that

$$
\frac{l_{2}}{l_{1}}=\frac{\bar{l}_{2}}{\bar{l}_{1}}, \frac{l_{3}}{l_{1}}=\frac{\bar{l}_{3}}{\bar{l}_{1}}
$$

Note that

$$
\begin{aligned}
\bar{l}_{1}^{2} & =\left(\bar{u}_{2}-\bar{u}_{1}\right)^{2}+\left(\bar{v}_{2}-\bar{v}_{1}\right)^{2}=\left(1+k^{2}\right)\left(\bar{v}_{2}-\bar{v}_{1}\right)^{2} \\
& =\frac{4\left(b^{2} k^{2}-\left(1+k^{2}\right)\left(b^{2}-r^{2}\right)\right)}{\left(1+k^{2}\right)}
\end{aligned}
$$




$$
\begin{aligned}
\bar{l}_{3}^{2} & =\left(\bar{u}_{4}-\bar{u}_{3}\right)^{2}+\left(\bar{v}_{4}-\bar{v}_{3}\right)^{2} \\
& =\frac{4\left(\left(b k-x_{2}\right)^{2}-\left(1+k^{2}\right)\left(b^{2}-r^{2}\right)\right)}{\left(1+k^{2}\right)}
\end{aligned}
$$

So $\left(\frac{l_{3}}{l_{1}}\right)^{2}=\left(\frac{\bar{l}_{3}}{l_{1}}\right)^{2}$ and $\left(\frac{l_{2}}{l_{1}}\right)^{2}=\left(\frac{\bar{l}_{2}}{l_{1}}\right)^{2}$ lead to

$b^{2}-\left(1+k^{2}\right) r^{2}+\frac{2 b k x_{2}-x_{2}^{2}}{\left(1-\frac{l_{3}^{2}}{l_{1}^{2}}\right)}=0$

$$
b^{2}-\left(1+k^{2}\right) r^{2}+\frac{2 b k x_{2}-x_{2}^{2}}{\left(1-\frac{l_{2}^{2}}{l_{1}^{2}}\right)}=0
$$

The denominators of (4.6) and (4.7) are non-zero with probability one as shown in Lemma 4.1. By subtracting (4.7) from (4.6) and the assumption that $x_{2} \neq 0$,

$$
b k=\frac{1}{2} x_{2}
$$

By plugging (4.8) back into any one of (4.7) and (4.6), we have

$$
\begin{gathered}
b^{2}-r^{2}\left(1+k^{2}\right)=0 \Rightarrow \\
b= \pm r \sqrt{1+k^{2}}
\end{gathered}
$$

and

$$
\left(\frac{1}{2} x_{2}\right)^{2}=\left( \pm r k \sqrt{1+k^{2}}\right)^{2} .
$$

(4.10) is a polynomial equation in $k$ and has finite number of solutions by the fundamental theorem of algebra [16]. Consequently, from (4.8), $b$ also has a finite number of solutions. This completes the proof.

We now turn to the proof of Theorem 4.1.

Proof: of Theorem 4.1. First, an arbitrary line $\alpha x+\beta y+\gamma=$ 0 can take two forms, $x=c$ or $y=k x+b$. However, to show uniqueness of the line if it intersects with the sensing region of three circles with non-collinear centers, the proofs for the cases $x=c$ and $y=b$ are the same by rotating the coordinate by 90 degree. So we focus on the line $y=k x+b$ as described in Lemmas 4.1 and 4.2. Suppose that a line $\bar{L}$ intersects three circles. Then, from Lemmas 4.1 and 4.2 , both $L$ and $\bar{L}$ must be from the set of finite candidate lines intersecting the first two circles with the fixed length ratios $\frac{l_{i}}{l_{1}}, i \in\{2,3\}$. Let $L$ and $\bar{L}$ intersect the third circle at $\left(\begin{array}{c}v_{i} \\ u_{i}\end{array}\right)$ and $\left(\begin{array}{c}\bar{v}_{i} \\ \bar{u}_{i}\end{array}\right), i \in\{5,6\}$ respectively. Further, for $i \in\{4,5\}$, suppose

$$
l_{i}=\left\|\left(\begin{array}{c}
v_{i+1} \\
u_{i+1}
\end{array}\right)-\left(\begin{array}{c}
v_{i} \\
u_{i}
\end{array}\right)\right\|, \bar{l}_{i}=\left\|\left(\begin{array}{c}
\bar{v}_{i+1} \\
\bar{u}_{i+1}
\end{array}\right)-\left(\begin{array}{c}
\bar{v}_{i} \\
\bar{u}_{i}
\end{array}\right)\right\| .
$$

as shown in Figure 2 and Figure 5. For each $\bar{L}$ from the (finite) candidate set, the slope and intercept $\left(k_{i}, b_{i}\right)$ are given. To satisfy the additional length ratios, if the line intersects the third circle,

$$
\frac{l_{4}}{l_{1}}=\frac{\bar{l}_{4}}{\bar{l}_{1}}, \frac{l_{5}}{l_{1}}=\frac{\bar{l}_{5}}{\bar{l}_{1}}
$$

the center of the third circle $\left(x_{3}, y_{3}\right)$ cannot be arbitrary but must satisfy the above fixed ratios (4.11). By the exact arguments as in the Lemma 4.2 , it can be shown that this results in a finite order polynomial equation in one of the unknowns $x_{3}$ or $y_{3}$. Again by the fundamental theorem of algebra that a finite order non-constant single variable polynomial equation has finite solutions [16], there are only a finite number of solutions $x_{3}$ for each candidate $\bar{L}$ line. Therefore, given the first two centers and the length ratios, there are only a finite number of the third centers that would allow a line $\bar{L}$ to have the same length ratios as the line $L$. Generically placed sensors will preclude the third center from coinciding exactly with these finitely many isolated points. This completes the proof.

Unsurprisingly, the above results can be extended if the line $L$ intersects more than 3 circles with known length ratios.

\subsection{Algorithm with unknown speed}

To apply the algorithm implied in section 4, estimates of the unknown speed $s$ and the chord length $d_{i}$ have to be calculated. Since the speed $s$ is unknown, we may replace $\sqrt{r^{2}-d_{i}^{2}}$ by $\sqrt{r^{2}-\hat{d}_{i}^{2}(\hat{s})}$ where $\hat{s}$ is an estimate of $s$ and the $\hat{d}_{i}(\hat{s})$ are the estimates of $d_{i}$,

$$
\hat{d}_{i}=\frac{\tau_{i 2}-\tau_{i 1}}{2} \hat{s}
$$

Clearly, if $\hat{s}=s$, one of the four solutions from

$$
\left[\begin{array}{lll}
x_{1} & y_{1} & 1 \\
x_{2} & y_{2} & 1 \\
x_{3} & y_{3} & 1
\end{array}\right]\left[\begin{array}{l}
\alpha \\
\beta \\
\gamma
\end{array}\right]=\left[\begin{array}{l} 
\pm \sqrt{r^{2}-\hat{d}_{1}^{2}} \\
\pm \sqrt{r^{2}-\hat{d}_{2}^{2}} \\
\pm \sqrt{r^{2}-\hat{d}_{3}^{2}}
\end{array}\right]
$$

satisfies $\alpha^{2}(\hat{s})+\beta^{2}(\hat{s})=1$. However, there is a fundamental difference between the cases of known and unknown speed. In the case of known speed, the length ratios $\frac{l_{i}}{l_{1}}$ do not play any role in ensuring the uniqueness of the line while in the unknown speed case, these ratios are necessary. These ratios can be divided into two types, the chord ratios $\frac{\tau_{i 2}-\tau_{i 1}}{\tau_{12}-\tau_{11}}$, and 
the outer length ratios $\frac{\tau_{(i+1) 1}-\tau_{i 2}}{\tau_{12}-\tau_{11}}$. The chord ratios are taken care of automatically in (4.12) because $\frac{\hat{d}_{i}}{\hat{d}_{1}}=\frac{\tau_{(i+1) 1}-\tau_{i 2}}{\tau_{12}-\tau_{11}}$. So the algorithm has to account for the outer length ratios. We replace (3.2) and (3.3) in section 3 with (4.13) and (4.14):

$$
\begin{gathered}
\min _{\hat{s}>0}\left(\alpha^{2}(\hat{s})+\beta^{2}(\hat{s})-1\right)^{2} \\
\text { s.t. }\left[\begin{array}{lll}
x_{1} & y_{1} & 1 \\
x_{2} & y_{2} & 1 \\
x_{3} & y_{3} & 1
\end{array}\right]\left[\begin{array}{l}
\alpha \\
\beta \\
\gamma
\end{array}\right]=\left[\begin{array}{l} 
\pm \sqrt{r^{2}-\hat{d}_{1}^{2}} \\
\pm \sqrt{r^{2}-\hat{d}_{2}^{2}} \\
\pm \sqrt{r^{2}-\hat{d}_{3}^{2}}
\end{array}\right]
\end{gathered}
$$

and

$$
\begin{aligned}
\frac{l_{i}}{l_{1}} & =\frac{\bar{l}_{i}}{\bar{l}_{1}}, i \in\{2,4\} \\
\hat{d}_{i}(\hat{s}) & =\frac{\left(\tau_{i 2}-\tau_{i 1}\right) \hat{s}}{2}, i \in\{1,2,3\} .
\end{aligned}
$$

The algorithm can now be summarized as follows:

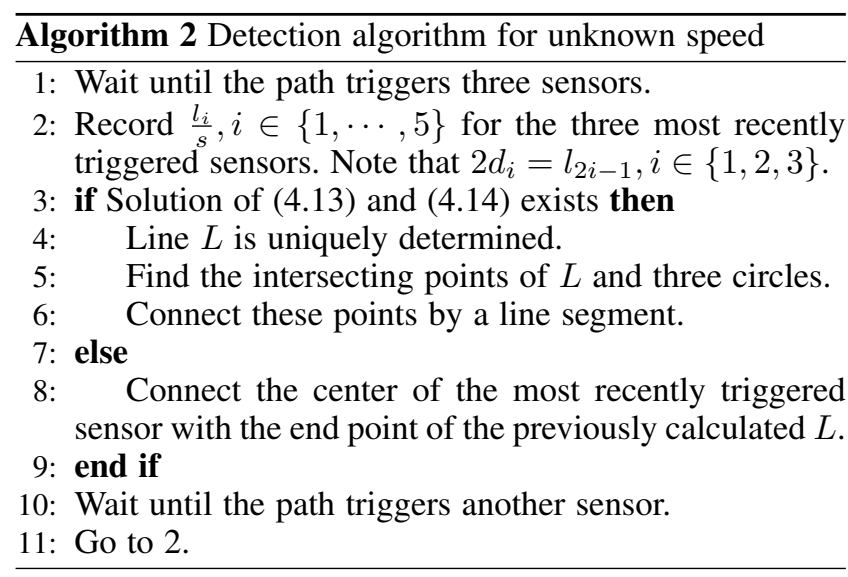

By the analysis in section 4 , the line $\alpha x+\beta y+\gamma=0$ can be uniquely determined for generic sensor placements.

\section{Robustness of proposed tracking algorithm to viola- tions of modeling assumptions}

We have focused in our analysis, for the sake of clarity, on a simple model of ideal error-free binary sensors with circular sensing areas and targets moving in piecewise linear trajectories. While these are reasonable approximations to important realistic applications, it is important to ensure that any practical tracking algorithm is not excessively sensitive to modeling assumptions. We now show that our algorithm is indeed robust to small deviations from ideal models. We formally show robustness to two different types of errors: first we consider calibration errors that result in the effective sensing range of different sensors being different from the nominal range and show that our algorithm can be readily adapted to deal with the resulting noisy sensor measurements. Next, we show that very similar methods can also be used to handle small deviations of the object trajectory from piecewise linear paths. While other types of impairment will require separate analyses, we further demonstrate the robustness of the proposed algorithm to these two and other types of sources of error numerically in Section 7.

\subsection{Uncertainty in the sensing range}

We now show how calibration uncertainties in the binary sensors can be accommodated in our approach.

Assumption 5.1 The sensing range of the $i$-th sensor is $r_{i}=$ $r+\Delta_{i},\left|\Delta_{i}\right| \leq \Delta<r$ where the $\Delta_{i}$ are iid random variables distributed in $-\Delta \leq \Delta_{i} \leq \Delta$ for some $\Delta \geq 0$.

Assume $\Delta=\alpha r$ depending on the quality of the sensors. For example, $\Delta=0.1 r$ which implies the uncertainty level $2 \Delta$ is about $20 \%$ of the nominal value $r$. Now, let us reconsider (4.13). Clearly, if the sensing range $r$ is accurate and $s$ is available, for generic sensor locations, only one solution of $A_{3}[\alpha, \beta, \gamma]^{T}=e_{3}$ i.e. $[\hat{\alpha}, \hat{\beta}, \hat{\gamma}]^{T}=\left(A_{3}^{T} A_{3}\right)^{-1} A_{3}^{T} e_{3}$ would satisfy $\hat{\alpha}^{2}+\hat{\beta}^{2}=1$ obeyed by the line. With uncertainty, this is no longer valid. Since the actual radius $r_{i}=r+\Delta_{i}$ is unknown, the resultant chord length $\hat{d}_{i}$ could be larger than the assumed radius $r$ or equivalently $\sqrt{r^{2}-\hat{d}_{i}^{2}}$ could be a complex number. In such a case, the equation in solving $(\alpha, \beta, \gamma)$ is rendered meaningless. Thus in the presence of uncertainty, not every sensor provides useful information. To this end, we only use data from the $i$-th sensor if $r-\Delta>\hat{d}_{i}$ which guarantees $\left(r+\Delta_{i}\right)^{2}-\hat{d}_{i}^{2}>0$. Define

$A_{m}=\left[\begin{array}{ccc}x_{1} & y_{1} & 1 \\ x_{2} & y_{2} & 1 \\ \vdots & \vdots & \vdots \\ x_{m} & y_{m} & 1\end{array}\right], e_{m}\left(\hat{s}, \Delta_{i}\right)=\left[\begin{array}{c} \pm \sqrt{\left(r+\Delta_{1}\right)^{2}-\hat{d}_{1}^{2}} \\ \pm \sqrt{\left(r+\Delta_{2}\right)^{2}-\hat{d}_{2}^{2}} \\ \vdots \\ \pm \sqrt{\left(r-\Delta_{m}\right)^{2}-\hat{d}_{m}^{2}}\end{array}\right]$,

and $r-\Delta>\hat{d}_{i}, i \in\{1, \cdots, m\}, m \geq 3$

Now consider

$$
\left[\begin{array}{ccc}
x_{1} & y_{1} & 1 \\
x_{2} & y_{2} & 1 \\
\vdots & \vdots & \vdots \\
x_{m} & y_{m} & 1
\end{array}\right]\left[\begin{array}{l}
\alpha \\
\beta \\
\gamma
\end{array}\right]=\left[\begin{array}{c} 
\pm \sqrt{r^{2}-\hat{d}_{1}^{2}} \\
\pm \sqrt{r^{2}-\hat{d}_{2}^{2}} \\
\vdots \\
\pm \sqrt{r^{2}-\hat{d}_{m}^{2}}
\end{array}\right] \Rightarrow
$$




$$
\begin{aligned}
A_{m}[\alpha, \beta, \gamma]^{T} & =e_{m}\left(\hat{s}, \Delta_{i}\right)+ \\
& \underbrace{\left[\begin{array}{c} 
\pm \sqrt{r^{2}-\hat{d}_{1}^{2}} \\
\pm \sqrt{r^{2}-\hat{d}_{2}^{2}} \\
\vdots \\
\pm \sqrt{r^{2}-\hat{d}_{m}^{2}}
\end{array}\right]-\left[\begin{array}{c} 
\pm \sqrt{\left(r+\Delta_{1}\right)^{2}-\hat{d}_{1}^{2}} \\
\pm \sqrt{\left(r+\Delta_{2}\right)^{2}-\hat{d}_{2}^{2}} \\
\vdots \\
\pm \sqrt{\left(r+\Delta_{i}\right)^{2}-\hat{d}_{m}^{2}}
\end{array}\right]}_{\Delta e_{m}\left(\hat{s}, \Delta_{i}\right)}
\end{aligned}
$$

Obviously, the first part

$$
A_{m}[\alpha, \beta, \gamma]^{T}=e_{m}\left(\hat{s}, \Delta_{i}\right)
$$

is the equation had the uncertainties $\Delta_{i}$ been available and its solution $[\alpha, \beta, \gamma]^{T}=\left(A_{m}^{T} A_{m}\right)^{-1} A_{m}^{T} e_{m}\left(\hat{s}, \Delta_{i}\right)$ leads to the line $\bar{\alpha} x+\bar{\beta} y+\bar{\gamma}=0$ provided that $\bar{\alpha}^{2}+\bar{\beta}^{2}=1$ and the length ratios $\frac{l_{i}}{l_{1}}=\frac{\bar{l}_{i}}{l_{1}}$ are satisfied as in (4.13). The problem is that the $\Delta_{i}$ are unknown. To study the effect of the uncertainty $\Delta e_{m}\left(\hat{s}, \Delta_{i}\right)$, note that under the assumption that $r-\Delta>\hat{d}_{i}$, it is easily verified that $\sqrt{r^{2}-\hat{d}_{i}^{2}}-$ $\sqrt{\left(r+\Delta_{i}\right)^{2}-\hat{d}_{i}^{2}}=-\frac{\Delta_{i}}{\sqrt{1-\left(\frac{d_{i}}{r}\right)^{2}}}+$ h.o.t. where h.o.t. refers to higher order terms in $\Delta_{i}$. Obviously, if we consider the dominant term, i.e., the first order term, the effect of the uncertainty

$$
\left(A_{m}^{T} A_{m}\right)^{-1} A_{m}^{T}\left[\begin{array}{c}
-\frac{\Delta_{1}}{\sqrt{1-\left(\frac{d_{1}}{r}\right)^{2}}} \\
\vdots \\
-\frac{\Delta_{m}}{\sqrt{1-\left(\frac{d_{m}}{r}\right)^{2}}}
\end{array}\right]
$$

goes to zero as $m$ increases provided that only the good information $r-\Delta>\hat{d}_{i}$ 's, $i \in\{1,2, \ldots, m\}$ are used and $\Delta_{i}$ are zero mean. To summarize, we have Theorem 5.1.

Theorem 5.1 Suppose the conditions of Theorem 4.1 hold except that the circles have radii $r+\Delta_{i}$. Suppose $[\bar{\alpha}, \bar{\beta}, \bar{\gamma}]^{T}$ is the true solution and $[\hat{\alpha}, \hat{\beta}, \hat{\gamma}]^{T}$ denotes the estimate generated by Algorithm 2. Then the following hold:

- Assume $r-\Delta>\hat{d}_{i}, i \in\{1,2, \ldots, m\}$ and $\Delta_{i}$ is iid of zero mean. Then as $m$ increases

$$
\begin{aligned}
& {[\bar{\alpha}-\hat{\alpha}, \bar{\beta}-\hat{\beta}, \bar{\gamma}-\hat{\gamma}]^{T}=\left(A_{m}^{T} A_{m}\right)^{-1} A_{m}^{T} \times} \\
& {\left[\begin{array}{c}
-\frac{\Delta_{1}}{\sqrt{1-\left(\frac{d_{1}}{r}\right)^{2}}} \\
\vdots \\
-\frac{\Delta_{m}}{\sqrt{1-\left(\frac{d_{m}}{r}\right)^{2}}}
\end{array}\right]+\text { h.o.t. }} \\
& \rightarrow \text { h.o.t. }
\end{aligned}
$$

- Further if the center coordinates $\left(x_{i}, y_{i}\right)$ are iid uniformly distributed of zero mean, then as $m$ increases

$$
[\bar{\alpha}-\hat{\alpha}, \bar{\beta}-\hat{\beta}, \bar{\gamma}-\hat{\gamma}]^{T} \rightarrow[0,0, \text { h.o.t. }]^{T}
$$

Proof: The first part is a direct consequence of the discussion preceding Theorem 5.1. To show the second part, note that

$$
\begin{aligned}
& {[\bar{\alpha}-\hat{\alpha}, \bar{\beta}-\hat{\beta}, \bar{\gamma}-\hat{\gamma}]^{T}=\left(A_{m}^{T} A_{m}\right)^{-1} A_{m}^{T} \Delta e_{m}\left(\hat{s}, \Delta_{i}\right)} \\
& \rightarrow \operatorname{diag}\left\{\frac{1}{\sigma_{x}^{2}}, \frac{1}{\sigma_{y}^{2}}, 1\right\} \times \frac{1}{m}\left[\begin{array}{cccc}
x_{1} & x_{2} & \cdots & x_{m} \\
y_{1} & y_{2} & \cdots & y_{m} \\
1 & 1 & \cdots & 1
\end{array}\right] \Delta e_{m}\left(\hat{s}, \Delta_{i}\right)
\end{aligned}
$$

where $\sigma_{x}^{2}$ and $\sigma_{y}^{2}$ are the variances of $x_{i}$ and $y_{i}$. The conclusion follows from the fact that $x_{i}$ and $y_{i}$ are iid and zero mean. This completes the proof.

\subsection{Trajectories that are not precisely piecewise linear}

We now show how our algorithm can be adapted to work with object trajectories that are not precisely piecewise linear, possibly due to lack of smoothness. We have stated earlier that piecewise linear motion trajectories are a good approximation for the motion of objects in many practically important applications e.g. container trucks in a port. Of course, the motions of practical objects are never exactly linear. We now show in detail the specific challenges that small deviations from the piecewise linear motion presents for the tracking problem and how we can adapt certain details of our algorithm while retaining its core features, to accommodate such deviations. Specifically, let us consider one segment of object trajectory $L: \mathbf{z}(t)$ that can be approximated by a straight-line constant speed trajectory $L^{\prime}: \tilde{\mathbf{z}}(t)$ as formalized below:

Assumption 5.2 At all times, the trajectory satisfies $\| \mathbf{z}(t)$ $\tilde{\mathbf{z}}(t) \| \leq \epsilon$ for some $0 \leq \epsilon \ll r$. 
Note that we have assumed that the parameter $\epsilon$ that represents the size of the deviations of the trajectory from the linear motion approximation is small compared to the sensing range $r$ of the binary sensors. We now consider how an object moving with such a trajectory interacts with a single ideal binary sensor. Compared to ideal linear motion, we now have to deal with two complicating issues:

(1) The object can enter and exit the sensing range of the sensor more than once. Indeed, the object can potentially enter and exit the sensing region of the ideal sensor an arbitrarily large number of times, by "pingponging" back and forth rapidly while still satisfying Assumption 5.2.

(2) Even if an object only enters and exits the sensing range of the sensor only once, the resulting time-stamps only give us a noisy estimate of the corresponding chordlength.

We now show that (a) by using a simple hysteresis mechanism for identifying and ignoring unreliable observations, we can avoid the ping-ponging problem, and (b) the residual estimation error in estimated chord-lengths can be handled in exactly the same way as we handled the uncertainty in sensing range in Section 5.1. We assume that the nominal speed of the object $s$ is known or can be estimated.

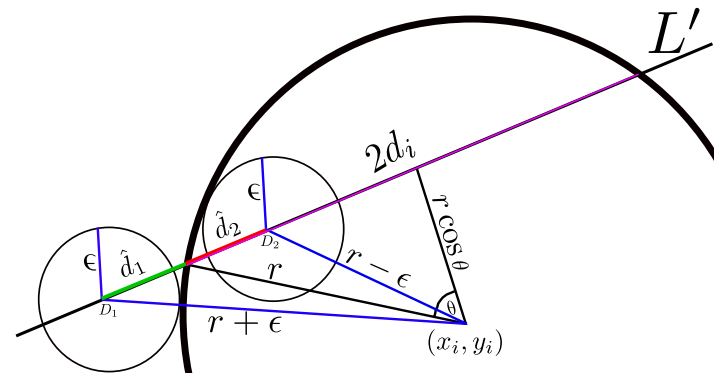

Fig. 6. Quantifying the time-stamp error due to trajectory that is not piecewise linear.

Consider a fictitious object moving with the known constant speed $s$ along the straight-line approximation $L^{\prime}$ to the actual trajectory $L$. Our approach is to attempt to recover this fictitious object's linear motion trajectory; Assumption 5.2 then guarantees that if we can accurately estimate this fictious trajectory $L^{\prime}$, our estimate will be close (within $\epsilon$ ) to the actual object's trajectory $L$. Consider Figure 6 that illustrates the intersection of a possibly non-smooth trajectory with the sensing area of one ideal binary sensor $i$. Let $\theta$ be the angle of intersection of the fictitious trajectory with the circle representing the sensing region as shown in Figure 6. While the object may enter and exit the sensing region multiple times, we note from Assumption 5.2, all entry and exit events associated with one approximately linear segment of the object's trajectory must occur within a total time interval no greater than $T_{\max }=\frac{2 r+2 \epsilon}{s}$. We then adopt the following simple convention: when an object first enters a sensor's range, we record all subsequent entry and exit events for the next $T_{\max }$ seconds and discard all events except the earliest entry and latest exit within this interval. We denote the time-stamps of these events $\tau_{i 1}$ and $\tau_{i 2}$ respectively. Let $\tau_{i 1}^{\prime}, \tau_{i 2}^{\prime}$ denote the time-stamps corresponding to the intersection of the fictitious straight line trajectory $L^{\prime}$ with the sensing region. We have the following result.

Lemma 5.1 The errors $\Delta \tau_{i 1} \doteq \tau_{i 1}-\tau_{i 1}^{\prime}, \Delta \tau_{i 2} \doteq \tau_{i 2}-$ $\tau_{i 2}^{\prime}$ in the entry and exit time-stamps are each bounded as follows:

$$
\left|\Delta \tau_{i 1}\right|,\left|\Delta \tau_{i 2}\right| \leq \frac{4 \epsilon}{s \sin \theta}
$$

Proof: Consider the entry time-stamp. At the instant $\tau_{i 1}$ when the object first enters the sensor $i$ 's sensing region, Assumption 5.2 guarantees that the fictitious object traveling along the linear motion trajectory $L^{\prime}$ must be somewhere between the points identified as $D_{1}$ and $D_{2}$ in Figure 6 . Therefore the fictitious object's entry into the sensing region cannot occur later than $\tau_{i_{1}}^{\prime} \leq \tau_{i 1}+\frac{\hat{d}_{1}}{s}$ nor earlier than $\tau_{i_{1}}^{\prime} \geq \tau_{i 1}-\frac{\hat{d}_{2}}{s}$. Thus we have

$$
\begin{gathered}
-\frac{\hat{d}_{2}}{s} \leq \tau_{i_{1}}^{\prime}-\tau_{i_{1}} \leq \frac{\hat{d}_{1}}{s} \\
\left\|\tau_{i_{1}}^{\prime}-\tau_{i_{1}}\right\| \leq \frac{\hat{d}_{1}+\hat{d}_{2}}{s}
\end{gathered}
$$

From Figure 6, standard geometric arguments give

$$
\begin{aligned}
\hat{d}_{1} & =\sqrt{(r+\epsilon)^{2}-r^{2} \cos ^{2} \theta}-\sqrt{r^{2}-r^{2} \cos ^{2} \theta} \\
& =\frac{(r+\epsilon)^{2}-r^{2}}{\sqrt{(r+\epsilon)^{2}-r^{2} \cos ^{2} \theta}+\sqrt{r^{2}-r^{2} \cos ^{2} \theta}} \\
& \leq \frac{(r+\epsilon)^{2}-r^{2}}{\sqrt{r^{2}-r^{2} \cos ^{2} \theta}}=\frac{(r+\epsilon)^{2}-r^{2}}{r \sin \theta} \\
\hat{d}_{2} & =\sqrt{r^{2}-r^{2} \cos ^{2} \theta}-\sqrt{(r-\epsilon)^{2}-r^{2} \cos ^{2} \theta} \\
& =\frac{r^{2}-(r-\epsilon)^{2}}{\sqrt{r^{2}-r^{2} \cos ^{2} \theta}+\sqrt{(r-\epsilon)^{2}-r^{2} \cos ^{2} \theta}} \\
& \leq \frac{r^{2}-(r-\epsilon)^{2}}{\sqrt{r^{2}-r^{2} \cos ^{2} \theta}}=\frac{r^{2}-(r-\epsilon)^{2}}{r \sin \theta}
\end{aligned}
$$

From $(5.19,5.20)$, we get

$$
\hat{d}_{1}+\hat{d}_{2} \leq \frac{(r+\epsilon)^{2}-(r-\epsilon)^{2}}{r \sin \theta}=\frac{4 \epsilon}{\sin \theta}
$$

The lemma for the entry time-stamp readily follows from (5.18) and (5.21). Similar arguments apply for the exit timestamp.

Equation (5.17) suggests that when $\sin \theta \approx 0$, the measured time-stamps can be extremely unreliable. This makes intuitive sense: consider the two cases as shown in Figure 7. 
In case $2, \theta<\cos ^{-1}\left(1-\frac{\epsilon}{r}\right)$, and we can see that it is in fact impossible to unambiguously identify "entry" and "exit" events for the object into the sensing area. For our tracking algorithm, we propose the simple strategy of discarding such observations. More specifically, suppose that we desire our time-stamps to be accurate to a maximum error of $\tau_{M A X}$, i.e. we wish to ensure that $\left\|\tau_{i_{1}}^{\prime}-\tau_{i_{1}}\right\| \leq \tau_{M A X}$ for some desired precision level $\tau_{M A X}$. From Lemma 5.1, this can be guaranteed by making sure that all observations used for the tracking algorithm satisfy $\theta>\theta_{M I N}=\sin ^{-1}\left(\frac{4 \epsilon}{s \tau_{M A X}}\right)$. This in turn can be guaranteed by making sure that all observed chord lengths satisfy $2 d_{i}>2 r \sin \theta_{M I N}=\frac{8 r \epsilon}{s \tau_{M A X}}$.
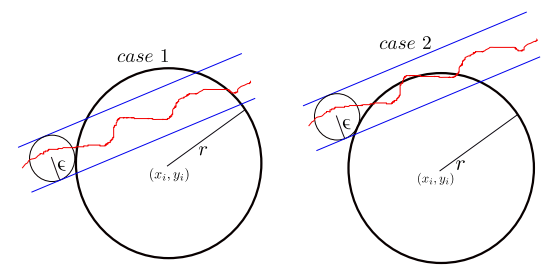

Fig. 7. Effect of a trajectory that is not piecewise linear: in case 1 , meaningful entry and exit time-stamps can be obtained, in case 2 , the sensor observations are unreliable.

We now show how timing uncertainties from trajectories that are not precisely piecewise linear, can be accommodated robustly in our approach. Observe that the error introduced in $d_{i}, i \in\{1,2,3\}$ and by extension $l_{i}, i \in\{1, \cdots, 2 m-1\}$ for $m$ sensors can be analyzed in the same manner as the uncertainty in the nominal sensing range $r$ was treated in section 5.1. We alter Assumption 5.1 to Assumption 5.3 below.

Assumption 5.3 The half chord length of the $i$-th sensor is $\hat{d}_{i}=d_{i}+\Delta_{d_{i}},\left|\Delta_{d_{i}}\right| \leq s \tau_{M A X}$ where the $\Delta_{d_{i}}$ are modeled as zero mean random variables.

It is seen that similar to the case where the error was in the sensing range, we use data which meet the criteria $r-\Delta_{d}>$ $d_{i}$.

Now consider

$$
\left[\begin{array}{ccc}
x_{1} & y_{1} & 1 \\
x_{2} & y_{2} & 1 \\
\vdots & \vdots & \vdots \\
x_{m} & y_{m} & 1
\end{array}\right]\left[\begin{array}{l}
\alpha \\
\beta \\
\gamma
\end{array}\right]=\left[\begin{array}{c} 
\pm \sqrt{r^{2}-\hat{d}_{1}^{2}} \\
\pm \sqrt{r^{2}-\hat{d}_{2}^{2}} \\
\vdots \\
\pm \sqrt{r^{2}-\hat{d}_{m}^{2}}
\end{array}\right] \Rightarrow
$$

$$
\begin{aligned}
& A_{m}\left[\begin{array}{l}
\alpha \\
\beta \\
\gamma
\end{array}\right]= \underbrace{\left[\begin{array}{c} 
\pm \sqrt{r^{2}-d_{1}^{2}} \\
\pm \sqrt{r^{2}-d_{2}^{2}} \\
\vdots \\
\pm \sqrt{r^{2}-d_{m}^{2}}
\end{array}\right]}_{e_{m}\left(\hat{s}, \Delta_{d_{i}}\right)}+ \\
& \underbrace{\left[\begin{array}{c} 
\pm \sqrt{r^{2}-\left(d_{1}+\Delta_{d_{1}}\right)^{2}} \\
\pm \sqrt{r^{2}-\left(d_{2}+\Delta_{d_{2}}\right)^{2}} \\
\vdots
\end{array}\right]-\left[\begin{array}{c} 
\pm \sqrt{r^{2}-d_{1}^{2}} \\
\pm \sqrt{r^{2}-d_{2}^{2}} \\
\vdots \\
\pm \sqrt{r^{2}-\left(d_{m}+d_{m}^{2}\right.}
\end{array}\right]}_{\Delta e_{m}\left(\hat{s}, \Delta_{d_{i}}\right)}
\end{aligned}
$$

In the absence of uncertainties $\Delta_{d_{i}}$, (5.22) reduces to:

$$
A_{m}[\alpha, \beta, \gamma]^{T}=e_{m}\left(\hat{s}, \Delta_{d_{i}}\right)
$$

The solution of (5.23), which is

$$
[\bar{\alpha}, \bar{\beta}, \bar{\gamma}]^{T}=\left(A_{m}^{T} A_{m}\right)^{-1} A_{m}^{T} e_{m}\left(\hat{s}, \Delta_{d_{i}}\right)
$$

leads to the line $\bar{\alpha} x+\bar{\beta} y+\bar{\gamma}=0$ provided that $\bar{\alpha}^{2}+\bar{\beta}^{2}=1$ and the length ratios $\frac{l_{i}}{l_{1}}=\frac{\bar{l}_{i}}{l_{1}}$ are satisfied as in (4.13). We also note that under the assumption that $r-\Delta_{d}>d_{i}$, it is easily verified that

$$
\sqrt{r^{2}-\left(d_{i}+\Delta_{d_{i}}\right)^{2}}-\sqrt{r^{2}-d_{i}^{2}}=-\frac{\Delta_{d_{i}}}{\sqrt{\left(\frac{r}{d_{i}}\right)^{2}-1}}+\text { h.o.t. }
$$

where h.o.t. refers to higher order terms in $\Delta_{d_{i}}$. Obviously, if we consider the dominant term, i.e., the first order term, the effect of the uncertainty

$$
\left(A_{m}^{T} A_{m}\right)^{-1} A_{m}^{T}\left[\begin{array}{c}
-\frac{\Delta_{d_{1}}}{\sqrt{\left(\frac{r}{d_{1}}\right)^{2}-1}} \\
\vdots \\
-\frac{\Delta_{d_{m}}}{\sqrt{\left(\frac{r}{d_{m}}\right)^{2}-1}}
\end{array}\right]
$$

goes to zero as $m$ increases provided that only the good information $r-\Delta_{d}>d_{i}$ 's, $i \in\{1,2, \ldots, m\}$ are used and $\Delta_{d_{i}}$ are zero mean. To summarize, we have the following Theorem 5.2. 
Theorem 5.2 Suppose the conditions of Theorem 4.1 hold except that the observations have chord lengths $d_{i}+\Delta_{d_{i}}$. Suppose $[\bar{\alpha}, \bar{\beta}, \bar{\gamma}]^{T}$ is the true solution and $[\hat{\alpha}, \hat{\beta}, \hat{\gamma}]^{T}$ denotes the estimate generated by Algorithm 2. Then the following hold:

- Assume $r-\Delta_{d_{i}}>d_{i}, i \in\{1,2, \ldots, m\}$ and $\Delta_{d_{i}}$ is iid of zero mean. Then as $m$ increases

$$
\begin{gathered}
{[\bar{\alpha}-\hat{\alpha}, \bar{\beta}-\hat{\beta}, \bar{\gamma}-\hat{\gamma}]^{T}=\left(A_{m}^{T} A_{m}\right)^{-1} A_{m}^{T} \times} \\
{\left[\begin{array}{c}
-\frac{\Delta_{d_{1}}}{\sqrt{\left(\frac{r}{d_{1}}\right)^{2}-1}} \\
\vdots \\
\left.-\frac{\Delta_{d_{m}}}{\sqrt{\left(\frac{r}{d_{m}}\right)^{2}-1}}\right]
\end{array}\right]+\text { h.o.t. }} \\
\rightarrow \text { h.o.t. }
\end{gathered}
$$

- Further if the center coordinates $\left(x_{i}, y_{i}\right)$ are iid uniformly distributed of zero mean, then as $m$ increases

$$
[\bar{\alpha}-\hat{\alpha}, \bar{\beta}-\hat{\beta}, \bar{\gamma}-\hat{\gamma}]^{T} \rightarrow[0,0, \text { h.o.t. }]^{T}
$$

Proof: The proof follows the same argument as that in Theorem 5.1.

\section{Density of sensors needed for accurate tracking}

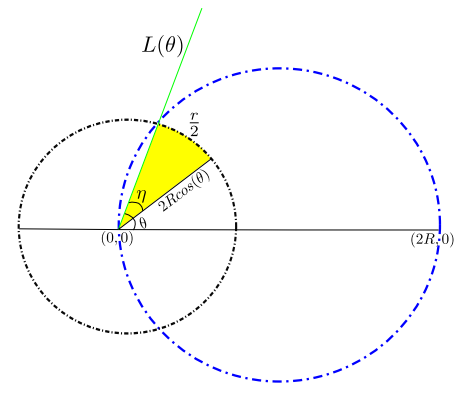

Fig. 8. Illustration of proof of Theorem 6.1

We now address the following question: Should the trajectory be a single straight line passing through $\mathcal{D}$, does its estimation require a high density of sensors in $\mathcal{D}$ ? We will show in this section that a high density is unnecessary, and that in fact vanishingly small densities also suffice. It is evident from the previous sections that should a line intersect the sensing ranges of three generically placed sensors, then it can be uniquely determined from the timestamps delineating entrance into and departure from these sensing ranges.

Thus we ask the following related question. Suppose the coverage area of the sensor network $\mathcal{D}$ is large compared to the sensing range of a sensor. Specifically suppose $\mathcal{D} \subset \mathbb{R}^{2}$ is a large disk with radius $R \gg r$. Asymptotically, what sensor density guarantees that this line intersects the sensing region of at least one of the sensors? Asymptotically, this density will be of the same order as that guaranteeing intersection with the sensing ranges of at least three sensors. We observe that the results we present extend readily to $\mathcal{D}$ that is rectangular or has other shapes.

We choose the origin and the coordinate axes so that the center of the disk $\mathcal{D}$ is at $(R, 0)$ as shown in Figure 8. Consider a line passing through the origin with an angle $\theta$ with respect to the horizontal axis, $L(\theta)$. Note that because of the circular symmetry of the problem, there is no loss of generality in our choice of line $L(\theta)$ to represent the trajectory of the object to be tracked.

Let the sensing range of the binary sensor $i$ be a circle of radius $r$ centered at $\left(x_{i}, y_{i}\right)$ uniformly distributed in $\mathcal{D}$. Let $N$ be the number of the binary sensors in $\mathcal{D}$ and $P(N, R, r, L(\theta))$ be the probability that the line $L(\theta)$ does not intersect any small circle of radius $r$ in $\mathcal{D}$. The probability is with respect to the uniform distribution defining sensor locations. What we are interested in is the asymptotic dependence of $P(N, R, r, L(\theta))$ on $R$. As will be evident in the sequel this allows us to draw conclusions about sensor densities.

Theorem 6.1 Let the centers $\left(x_{i}, y_{i}\right)$ be i.i.d uniformly distributed in

$$
\mathcal{D}=\left\{z \in \mathbb{R}^{2} \mid\left\|z-[R, 0]^{\top}\right\| \leq R\right\}
$$

Define $L(\theta)$ to be the line passing through the origin, making an angle $\theta$ with the $x$-axis. Assume $\theta \in(-\pi / 2, \pi / 2)$ and

$$
\frac{R}{r}=c_{1} N^{1-\delta}
$$

for some $c_{1}>0$ and $0<\delta<1$. Then,

$$
\lim _{N \rightarrow \infty} P(N, R, r, L(\theta))=0 .
$$

Proof: The chord length of the line $L(\theta)$ for $\theta \in$ $(-\pi / 2, \pi / 2)$ is $d=2 R \cos (\theta)$. Define the circle centered at the origin with radius $d$. Make an arc of the arc length $\frac{r}{2}$ along the circle toward the positive horizonal axis as shown in Figure 4. The angle $\eta$ and the area of the small fan-like sector in Figure 4 are

$$
\begin{aligned}
\eta & =\frac{r}{2} \frac{1}{2 \pi d} 2 \pi=\frac{1}{4 \cos (\theta)} \frac{r}{R}, \\
A(\theta, \eta) & =\frac{\eta}{2 \pi} \pi d^{2}=\frac{\cos (\theta)}{2} r R
\end{aligned}
$$

respectively. Clearly, if any of the $N$ sensors is placed in the fan-like sector, the circle intersects the line $L(\theta)$. Because of the uniform distribution and the fact that the fan-like sector 
is completely inside the big disk, the probability that the center of the $i$ th small circle $\left(x_{i}, y_{i}\right), 1 \leq i \leq N$, is in the fan-like sector is the ratio of the areas of the fan-like sector and the big disk $\frac{\cos (\theta)}{2} r R \frac{1}{\pi R^{2}}=\frac{\cos (\theta)}{2 \pi} \frac{r}{R}=c_{2} \frac{r}{R}$. So the probability that none of the centers of the $N$ small circles lies in the fan-like sector is for some $c_{3}$, and any $x \leq c_{3} N^{\delta}$

$$
\begin{aligned}
P(N, R, r, L(\theta)) & \leq\left(1-c_{2} \frac{r}{R}\right)^{N}=\left(1-c_{3} \frac{N^{\delta}}{N}\right)^{N} \\
& \leq\left(1-\frac{x}{N}\right)^{N} .
\end{aligned}
$$

As

$$
\lim _{N \rightarrow \infty}\left(1-\frac{x}{N}\right)^{N}=e^{-x},
$$

and $x>0$ can be arbitrarily large as $N \rightarrow \infty$, it follows that

$$
\lim _{N \rightarrow \infty} P(N, R, r, L(\theta))=0 .
$$

The following corollary that addresses the simultaneous intersection with $m$ sensing ranges, follows easily.

Corollary 6.1 Let $m$ be any fixed positive number and $P_{m}(N, R, r, L(\theta))$ the probability that the line $L(\theta)$ does not intersect $m$ or more small circles. Then, under the conditions of Theorem 6.1,

$$
\lim _{N \rightarrow \infty} P_{m}(N, R, r, L(\theta))=0 .
$$

Let us now address the implication to density. Suppose $\delta$ in Theorem 6.1 is less than 0.5 . Then the sensor density

$$
\rho=\frac{N}{\pi R^{2}}=O\left(\frac{1}{N^{1-2 \delta}}\right)
$$

declines to zero as $N$ goes to infinity. Thus the probability that a line $L(\theta)$ intersects at least one sensors sensing range approaches one even with vanishingly small densities. Some other remarks are in order

\section{Remark:}

- At first blush the problem we are considering appears to be related to the disk covering problem for finding the least number of small circles of radius $r$ for covering a disk of radius $R$. For small enough $r$, the least number $N$ of sensors whose sensing ranges collectively cover $\mathcal{D}$, satisfies [12]

$$
\frac{N \pi r^{2}}{\pi R^{2}}=\frac{2 \pi}{3 \sqrt{3}}
$$

According to this the total area covered by the sensors $N \pi r^{2}$ has to be larger than the area of the big disk $\pi R^{2}$ by a factor of $\frac{2 \pi}{3 \sqrt{3}}$. This result effectively demands that the sensing ranges be overlapping. By contrast our results do not require any such overlap, see e.g. Theorem 6.1. In fact suppose again that $0<\delta<0.5$. Then

$$
\frac{N \pi r^{2}}{\pi R^{2}}=\frac{1}{c_{1}^{2}} \cdot \frac{1}{N^{1-2 \delta}}
$$

approaches zero as $N$ approaches infinity. Thus, sensing ranges do not have to cover $\mathcal{D}$ and in fact the ratio of the total area covered by the $N$ sensors and the area of $\mathcal{D}$ can approach zero.

- Again almost all results in the binary sensor literature assume a very high density of sensors so that the whole area is completely covered by binary sensors. With a very high density, every point in the region lies in the sensing range of several sensors. The object position and trajectory can be pinned down with a higher degree of accuracy because the intersection is much smaller than the sensing area of any single sensor. Clearly, a higher density implies that more sensing ranges overlap resulting in a smaller area of intersection and thus a more accurate estimate. In fact, as shown in [5] the estimation accuracy is in the order of $1 / \rho r$. The estimation error goes to zero only when $\rho \rightarrow \infty$. If the density $\rho \rightarrow 0$, the estimation error goes to infinity. By contrast in our method, $\rho$ does not have to go to infinity to have an accurate estimate and can in fact be vanishingly small.

\section{Simulations}

\subsection{Tracking piecewise linear source trajectories with isotropic sensors}

We consider two cases, (1) the sensing radius $r$ is exactly known and (2) the actual sensing radius is unknown and random uniformly distributed in $[0.85 r, 1.5 r]$, representing a range of uncertainty that is $30 \%$ of the nominal range. In the latter case, the sensing ranges are iid random variables. Let $\mathcal{D} \subset \mathbb{R}^{2}$ be a square of area of $4 \mathrm{~km}^{2}$ centered at the origin.

The nominal sensing radius of the binary sensors is $r=$ $100 \mathrm{~m}$. Forty one sensors are uniformly distributed in $\mathcal{D}$. Note the area covered by the sensors is about $13 \%$ of the total area of $\mathcal{D}$ and thus $87 \%$ of the area was not covered. The unknown path of the object consists of 4 line segments with 4 different speeds in each segment as shown in Figure 9. The unknown path is in bold and the arrows represent the direction of movement. The solid dark line is the estimate of the path obtained by our algorithm in (4.13). Figure 9 depicts the tracking results when the sensing radius is known exactly and Figure 10 is with uncertain sensing ranges. Clearly in both cases, the unknown path is accurately estimated even when it changes the direction and speed several times. 


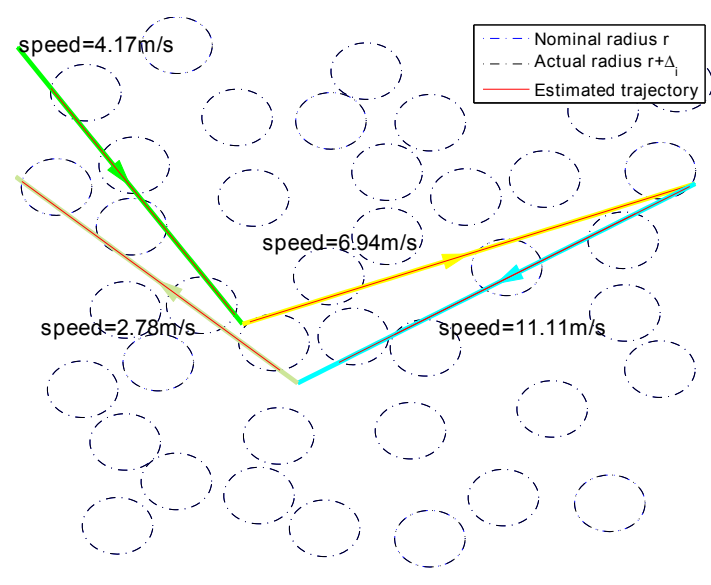

Fig. 9. No uncertainty in the sensing radius.

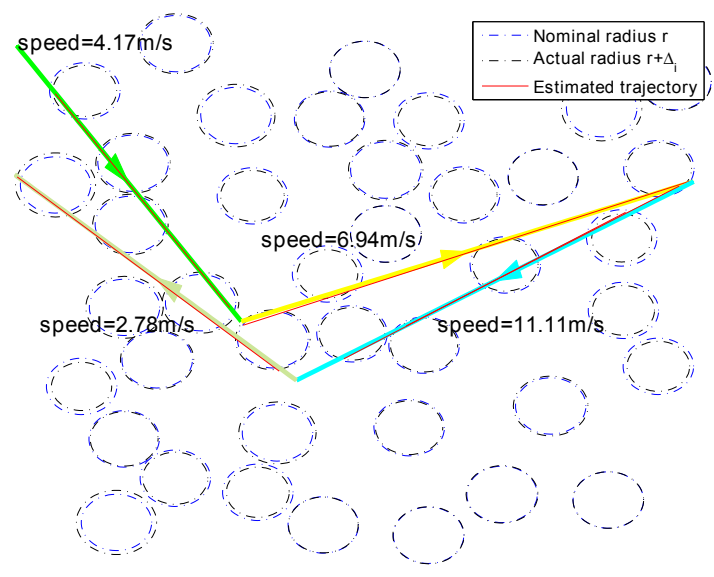

Fig. 10. Uncertainty in the sensing range.

\subsection{Tracking non-linear source trajectories with anisotropic sensors with timing jitters}

We consider sensors whose sensing ranges are anisotropic. In particular, the sensing range is modeled as ellipsoid with semi-major axis of $5 \mathrm{~m}$ and semi-minor axis as $4.5 \mathrm{~m}$. We further consider a non-linear source trajectory within a tube with $\epsilon \leq 0.5 \mathrm{~m}$ as discussed before. We also consider timing errors and jitter with non-deterministic additive white Gaussian noise $(\mathrm{AWGN}) \sim N(0,1 \mathrm{msec})$. The assumed nominal radius for the sensors is $5 \mathrm{~m}$. The sensors are located at $(55,-3),(70,4),(80,-2)$ and $(90,4)$ in $\mathbb{R}^{2}$. The ellipsoid sensing ranges are rotated by $\frac{-\pi}{4}, \frac{7 \pi}{36}, \frac{\pi}{36}$ and $\frac{21 \pi}{36}$ respectively to account for random geometric placement of the sensors. Under these conditions, the performance of our algorithm is shown in Figure 11. Clearly the unknown nonlinear path is accurately estimated by the linear estimate with the maximum error of the linear estimate from true nonlinear trajectory at any point in time being $0.7 \epsilon$.

\section{Concluding remarks}

We have presented a novel approach to tracking using a binary sensor network. Unlike previous methods, our approach

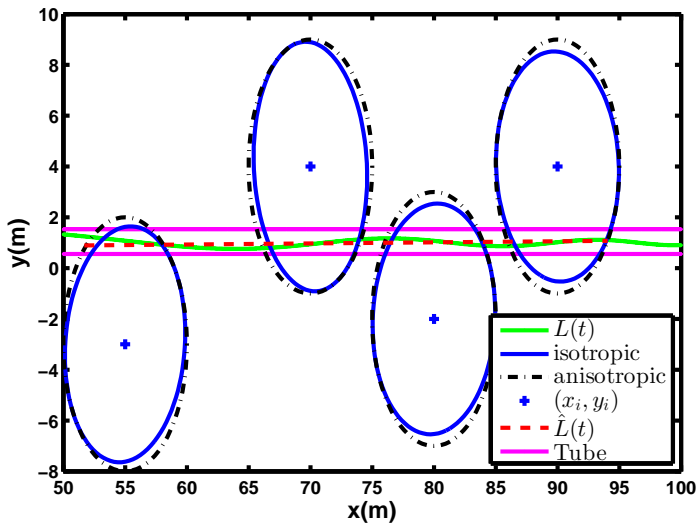

Fig. 11. Performance of algorithm for nonlinear trajectory, anisotropic sensors and timing jitters.

is not limited by the accuracy of individual sensors or the density of sensors, instead relying on the local smoothness of trajectories to achieve high tracking accuracy. We have presented a practical algorithm that works well in the presence of sensing errors embedding in sensing range, isotropic or otherwise and timing errors, and have demonstrated its efficacy using simulations. While we have considered piecewise linear trajectories, and presented simulations for a nonlinear trajectory within a tube of predefine width, the underlying ideas of our algorithm are quite general and can be extended to other classes of smooth trajectories that are of physical interest.

\section{Acknowledgements}

This work was in part supported by NSF grants CCF0830747, EPS-1101284, ECCS-1150801 and CNS1239509, DoE grant DE-FG52-09NA29364.

\section{References}

[1] R. Mudumbai and U. Madhow (2008), " Information theoretic bounds for sensor network localization”, IEEE ISIT, pp.1602-1606

[2] N. Patwari and A.O. Hero III (2003), "Using proximity and quantized RSS for sensor localization in wireless networks", Proceedings of the 2nd ACM international conference on Wireless sensor networks and applications, pp.20-29

[3] I.F. Akyildiz, W. Su, Y. Sankarasubramaniam and E. Cayirci (2002), "Wireless sensor networks: a survey", Computer networks, Vol.38, pp.393-422

[4] Z. Wang, E. Bulut and B.K. Szymanski (2010), "Distributed energy-efficient target tracking with binary sensor networks", ACM Transactions on Sensor Networks, Vol. 6, pp.32

[5] N. Shrivastava, R. Mudumbai, U. Madhow and S. Suri (2009), "Target tracking with binary proximity sensors", ACM Transactions on Sensor Networks, Vol. 5, pp.30

[6] J. Singh, U. Madhow, R. Kumar, S. Suri and R. Cagley (2007), "Tracking multiple targets using binary proximity sensors", Proceedings of the 6th international conference on Information processing in sensor networks, pp.529-538 
[7] T. Nguyen, D. Nguyen, H. Liu and D.A. Tran (2007), "Stochastic binary sensor networks for noisy environments", International Journal of Sensor Networks, Vol.2, pp.414-427

[8] W. Kim, K. Mechitov, J. Choi and S. Ham (2005), "On target tracking with binary proximity sensors", IEEE IPSN 2005, pp.301-308

[9] Q. Le and L.M. Kaplan (2010), "Target localization using proximity binary sensors", IEEE Aerospace Conference, pp.1-8

[10] M. Lázaro, M. Sánchez-Fernández and A. Artés-Rodríguez, (2009), "Optimal sensor selection in binary heterogeneous sensor networks", IEEE Trans on Signal Processing, Vol.57, pp.1577-1587

[11] B. F. La Scala, M.R. Morelande and C.O. Savage (2006), "Robust target tracking with unreliable binary proximity sensors", IEEE Processing ICASSP, Vol. 4, pp.IV

[12] R. Kershner (1939), "The number of circles covering a set", American Journal of Mathematics, pp.665-671

[13] P. Kump, E. Bai, K. Chan, B. Eichinger and K. Li (2012), "Variable selection via RIVAL (removing irrelevant variables amidst Lasso iterations) and its application to nuclear material detection", Automatica, Vol.48, pp.2107-2115

[14] N. Katenka, E. Levina and G. Michailidis (2008), "Robust target localization from binary decisions in wireless sensor networks", Technometrics, Vol. 50, pp.448-461

[15] R.A. Johnson (2013), “Advanced euclidean geometry", Courier Corporation

[16] B. Fine (1997), “The fundamental theorem of algebra”, Springer Science \& Business Media

[17] P.M. Djuric, M. Vemula and M.F. Bugallo (2008), “Target tracking by particle filtering in binary sensor networks", IEEE TRans on Signal Processing, Vol.56, pp.2229-2238

[18] Y. Busnel, L. Querzoni, R. Baldoni, M. Bertier and A. Kermarrec (2008), "On the deterministic tracking of moving objects with a binary sensor network", Distributed Computing in Sensor Systems, pp.46-59

[19] J. Aslam, Z. Butler, F. Constantin, V. Crespi, G. Cybenko and D. Rus (2003), "Tracking a moving object with a binary sensor network", ACM Proceedings of the 1st international conference on Embedded networked sensor systems, pp.150-161

[20] E. Xu, Z. Ding and S. Dasgupta (2013), "Target tracking and mobile sensor navigation in wireless sensor networks", IEEE Trans on Mobile Computing, Vol.12, pp.177-186

[21] I. Shames, S. Dasgupta, B. Fidan and D.O. Anderson (2012), "Circumnavigation using distance measurements under slow drift", IEEE Trans on Automatic Control, Vol.57, pp.889-903 